\title{
Scalar Diagnostics of the Inertial Measurement Unit
}

\author{
Vadim V. Avrutov \\ Instrument Making Faculty, National Technical University of Ukraine (Kiev Polytechnic Institute), Kiev, 03056, \\ Ukraine \\ E-mail: vyshgorod@gmail.com
}

\begin{abstract}
The scalar method of fault diagnosis systems of the inertial measurement unit (IMU) is described. All inertial navigation systems consist of such IMU. The scalar calibration method is a base of the scalar method for quality monitoring and diagnostics. Algorithms of fault diagnosis systems are developed in accordance with scalar calibration method. Algorithm verification is implemented in result of quality monitoring of IMU. A failure element determination is based in diagnostics algorithm verification and after that the reason of such failure is cleared. The process of verifications consists of comparison of the calculated estimations of biases, scale factor errors and misalignments angles of sensors to their data sheet certificate, which kept in internal memory of navigation computer. In result of such comparison the conclusion for working capacity of each one IMU sensor can be made and also the failure sensor can be determined.
\end{abstract}

Index Terms - Fault diagnosis, inertial measurement unit, inertial navigation systems, scalar calibration, quality monitoring and diagnostics.

\section{INTRODUCTION}

In recent years, the strong requirements for safety of autonomous vehicles caused new demands for reliability of the Inertial Navigation Systems and Inertial Measurement Units as their component parts. It is a reason for an increasing and expanding their testing program and using of fault diagnosis systems, which includes the capacity of detecting, isolating and identifying faults.

There are different methods of fault diagnosis of Inertial Navigation Systems (INS). More simple and wide application is monitoring of output level of signals of INS components parts made by technology of Built In Test Equipment (BITE) [1,2]. Besides, diagnostics could be made by multiple-choice alternative methods of optimal filtration [3,4] and functional diagnostic model methods [5]. If the functional diagnostic model methods are using for IMU and based on application of redundant or extra number of sensors, optimal filtration methods are using whole INS and required other information of instruments, which working on diverse from inertial technology principles (for example, information from satellite navigation receiver GPS/GLONASS or Doppler radar).
Above mentioned approaches are based on quantitative or numerical models, when using or generating signals that reflect inconsistencies between nominal and faulty system operation.

During the last time many investigations have been made using qualitative or analytical models, using neural networks [6, 7] and fuzzy logic techniques [8, 9].

From another side, it is known a scalar calibration method [10-12]. In that papers are described main features of a scalar calibration method for an inertial measurement unit consisting of gyroscopes and accelerometers. The method allows determine biases, scale factor errors and mounting misalignments of the sensors without applying special requirements for alignment of test equipment and sensors alignment on the test equipment. But it requires sufficiently high accuracy of measurement of the output signals of sensors: the algorithm works fine when the number of digits is at least eight decimal places in normalized output signals [12].

In this paper is suggested to use together scalar calibration method of gyroscopes and accelerometers [11] for fault diagnosis of IMU of Inertial Navigation Systems and also functional diagnostics model methods. But redundant sensors are not required for new method of scalar diagnostics. Normally it can use output signals of three gyros and three accelerometers only.

\section{Scalar Diagnostics ON THE Stationary BASE}

Let us consider IMU of strapdown INS [13] (Fig.1) consisting from a triad of single degree-of-freedom gyroscopes $G_{X}, G_{Y}, G_{Z}$ and a triad of accelerometers $A_{X}, A_{Y}, A_{Z}$ that are mounted to a vehicle with body frame oxyz with orthogonal sensitivity axes, as shown on Fig. 2.

Taking into consideration the errors of instruments (biases, scale-factor errors), mounting misalignments of the gyroscopes and accelerometers, which cause crosscoupling terms, and in-run random bias errors, the gyro's output signals may be expressed as shown below:

$$
\left[\begin{array}{c}
U_{\omega x} \\
U_{\omega y} \\
U_{\omega z}
\end{array}\right]=\left[\begin{array}{c}
B_{\omega x} \\
B_{\omega y} \\
B_{\omega z}
\end{array}\right]+\mathbf{K}_{\omega} \cdot\left[\begin{array}{c}
\omega_{x} \\
\omega_{y} \\
\omega_{z}
\end{array}\right]+\left[\begin{array}{c}
w_{\omega x} \\
w_{\omega y} \\
w_{\omega z}
\end{array}\right] .
$$




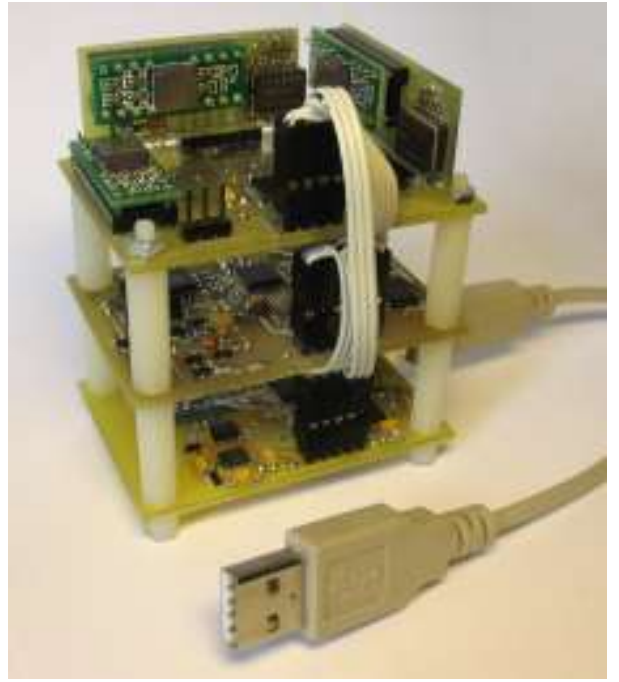

Fig.1. Inertial measurement unit with USB-port

Accelerometer's output signals also may be expressed as shown below:

$$
\left[\begin{array}{l}
U_{a x} \\
U_{a y} \\
U_{a z}
\end{array}\right]=\left[\begin{array}{c}
B_{a x} \\
B_{a y} \\
B_{a z}
\end{array}\right]+\mathbf{K}_{\mathbf{a}} \cdot\left[\begin{array}{l}
a_{x} \\
a_{y} \\
a_{z}
\end{array}\right]+\left[\begin{array}{c}
w_{a x} \\
w_{a y} \\
w_{a z}
\end{array}\right],
$$

where $U_{\omega(a) x}, U_{\omega(a) y}, U_{\omega(a) z}$ - is a set of output signals of gyroscopes (accelerometers), $\omega_{x}, \omega_{y}, \omega_{z}$ - are the applied angular rates acting about the principle axes of the vehicle, $a_{x}, a_{y}, a_{z}$ - are the accelerations acting along these same axes, $B_{\omega(a) x}, B_{\omega(a) y}, B_{\omega(a) z}$ - is a set of the residual fixed biases of gyroscopes (accelerometers), $w_{\omega(a) x}, w_{\omega(a) y}, w_{\omega(a) z}$ - is a set of the in-run random bias errors of gyroscopes (accelerometers),

$$
\begin{gathered}
\mathbf{K}_{\omega}=\left[\begin{array}{ccc}
S_{\omega x}+E_{\omega x} & S_{\omega x} \Delta_{x z} & -S_{\omega x} \Delta_{x y} \\
-S_{\omega y} \Delta_{y z} & S_{\omega y}+E_{\omega x} & S_{\omega y} \Delta_{y x} \\
S_{\omega z} \Delta_{z y} & -S_{\omega z} \Delta_{z y} & S_{\omega z}+E_{\omega x}
\end{array}\right], \\
\mathbf{K}_{\mathbf{a}}=\left[\begin{array}{ccc}
S_{a x}+E_{a x} & S_{a x} \Delta_{x z} & -S_{a x} \Delta_{x y} \\
-S_{a y} \Delta_{y z} & S_{a y}+E_{a x} & S_{a y} \Delta_{y x} \\
S_{a z} \Delta_{z y} & -S_{a z} \Delta_{z y} & S_{a z}+E_{a x}
\end{array}\right],
\end{gathered}
$$

$S_{\omega(a) x}, S_{\omega(a) y}, S_{\omega(a) z}$ - is the scale factors of gyroscopes (accelerometers), $E_{\omega(a) x}, E_{\omega(a) y}, E_{\omega(a) z}$ - is a set of the scale-factor errors of gyroscopes (accelerometers), $\Delta_{q j}{ }^{-}$ are the mounting misalignments angles or cross-coupling terms of gyroscopes (accelerometers) [2,11].

Here in notification of $\Delta_{x z}$ angle, the first index is shown that the unit is mounted on ox axes and has been rotated about $o z$ axes on $\Delta_{x z}$ angle.

Be noted that listed above linearized model (1) are present to closer for optical sensors like ring laser and fiber optic gyroscopes. For the conventional gyroscopes and dynamical tuned gyro, the above equations should be added to $3 \times 3$ matrixes representing the g-dependent bias coefficients and anisoelastic coefficients [2].

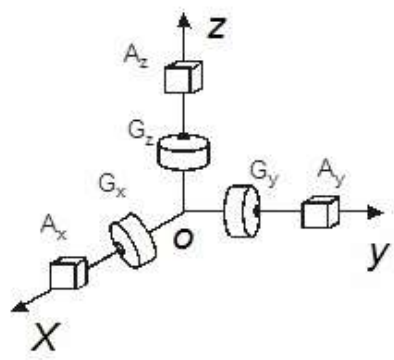

Fig.2. Inertial measurement unit

Let us assume that calibration of IMU has being done before and all above mentioned parameters like as residual fixed biases, scale-factor errors and mounting misalignments angles of gyroscopes and accelerometers are measured and reserved in internal INS computer's memory.

After that we will doing scalar diagnostics on fixed foundation in the gravity field of Earth, hence will pass from the body turn rate $\vec{\omega}$ to Earth's rate $\vec{\Omega}$ therefore the gyro's output signals (1) will be expressed as shown below and acceleration $\vec{a}$ to the projections of gravity vector $\vec{g}$ also accelerometer's output signals (2) will be expressed as shown below:

$U_{\Omega x}=B_{\omega x}+\left(S_{\omega x}+E_{\omega x}\right) \Omega_{x}+S_{\omega x} \Delta_{x z} \Omega_{y}-S_{\omega x} \Delta_{x y} \Omega_{z}+w_{\Omega x} ;$

$U_{\Omega y}=B_{\omega y}+\left(S_{\omega y}+E_{\omega y}\right) \Omega_{y}-S_{\omega y} \Delta_{y z} \Omega_{x}+S_{\omega y} \Delta_{y x} \Omega_{z}+w_{\Omega y}$;

$U_{\Omega z}=B_{\omega z}+\left(S_{\omega z}+E_{\omega z}\right) \Omega_{z}+S_{\omega z} \Delta_{z y} \Omega_{x}-S_{\omega z} \Delta_{z x} \Omega_{y}+w_{\Omega z}$,

$U_{g x}=B_{a x}+\left(S_{a x}+E_{a x}\right) g_{x}+S_{a x} \Delta_{x z} g_{y}-S_{a x} \Delta_{x y} g_{z}+w_{g x} ;$

$U_{g y}=B_{a y}+\left(S_{a y}+E_{a y}\right) g_{y}-S_{a y} \Delta_{y z} g_{x}+S_{a y} \Delta_{y x} g_{z}+w_{g y}$;

$U_{g z}=B_{a z}+\left(S_{a z}+E_{a z}\right) g_{z}+S_{a z} \Delta_{z y} g_{x}-S_{a z} \Delta_{z x} g_{y}+w_{g z}$,

Fig. 3 and Fig. 4 shows examples of output signals of gyroscopes and accelerometers of IMU with USB-port [13] on stationary base.
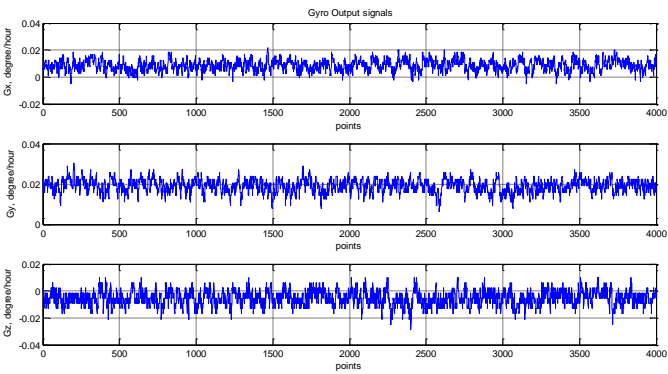

Fig.3. Output signals of gyroscopes ADXRS22295 (Gx and Gy) and ADXRS300 $(\mathrm{Gz})$ on stationary base.

I.J. Intelligent Systems and Applications, 2015, 11, 1-9 

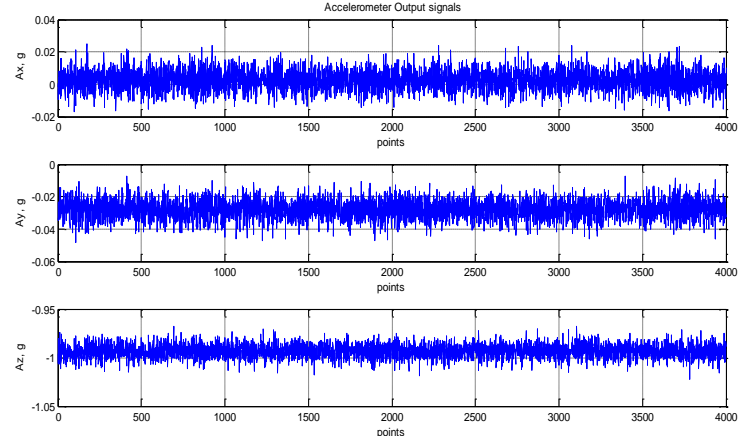

Fig.4. Output signals of accelerometers ADXL202 (Ax, Ay and Az) on stationary base.

Let's divide every expression of output signal of accelerometer on corresponding scale factor and vector's module $\vec{g}\left(g=\sqrt{g_{x}^{2}+g_{y}^{2}+g_{z}^{2}}\right)$ and every expression of output gyro signal on corresponding scale factor and vector's module $\vec{\Omega}\left(\Omega=\sqrt{\Omega_{x}^{2}+\Omega_{y}^{2}+\Omega_{z}^{2}}\right)$. Above two sets equations (3) and (4) are similar by form and therefore it will be enough to consider accelerometer's output signals (4) only after normalization:

$$
\begin{aligned}
& \frac{U_{g x}}{S_{a x} \cdot g}=\frac{B_{a x}}{S_{a x} \cdot g}+\left(1+\frac{E_{a x}}{S_{a x}}\right) \frac{g_{x}}{g}+\Delta_{x z} \frac{g_{y}}{g}-\Delta_{x y} \frac{g_{z}}{g}+\frac{w_{g x}}{S_{a x} \cdot g} \\
& \frac{U_{g y}}{S_{a y} \cdot g}=\frac{B_{a y}}{S_{a y} \cdot g}+\left(1+\frac{E_{a y}}{S_{a y}}\right) \frac{g_{y}}{g}-\Delta_{y z} \frac{g_{x}}{g}-\Delta_{y x} \frac{g_{z}}{g}+\frac{w_{g y}}{S_{a y} \cdot g} \\
& \frac{U_{g z}}{S_{a z} \cdot g}=\frac{B_{a z}}{S_{a z} \cdot g}+\left(1+\frac{E_{a z}}{S_{a z}}\right) \frac{g_{z}}{g}+\Delta_{z y} \frac{g_{x}}{g}-\Delta_{z x} \frac{g_{y}}{g}+\frac{w_{g z}}{S_{a z} \cdot g} .
\end{aligned}
$$

Let us to input new denotations of dimensionless output signals and values of right parts as follows:

$$
\begin{aligned}
& u_{g j}=\frac{U_{g j}}{S_{a j} g} ; \quad \bar{g}_{j}=\frac{g_{j}}{g} ; b_{g j}=\frac{B_{a j}}{S_{a j} g} ; e_{a j}=\frac{E_{a j}}{S_{a j}} ; n_{g j}=\frac{w_{g j}}{S_{a j} g} \\
& u_{\Omega j}=\frac{U_{\Omega j}}{S_{\omega j} \Omega} ; \bar{\Omega}_{j}=\frac{\Omega_{j}}{\Omega} ; \quad b_{\Omega j}=\frac{B_{\omega j}}{S_{\omega j} \Omega} ; e_{\omega j}=\frac{E_{\omega j}}{S_{\omega j}} ; n_{\Omega j}=\frac{w_{\Omega j}}{S_{\omega j} \Omega} .
\end{aligned}
$$

Here $j=x, y, z$.

Using above denotations (5) the normalized accelerometer's output signals can be described as

$$
\begin{aligned}
& u_{g x}=b_{g x}+\left(1+e_{a x}\right) \bar{g}_{x}+\Delta_{x z} \bar{g}_{y}-\Delta_{x y} \bar{g}_{z}+n_{g x} \\
& u_{g y}=b_{g y}+\left(1+e_{a y}\right) \bar{g}_{y}-\Delta_{y z} \bar{g}_{x}+\Delta_{y x} \bar{g}_{z}+n_{g y} \\
& u_{g z}=b_{g z}+\left(1+e_{a z}\right) \bar{g}_{z}+\Delta_{z y} \bar{g}_{x}-\Delta_{z x} \bar{g}_{y}+n_{g z} .
\end{aligned}
$$

After removal of brackets we will have

$$
\begin{aligned}
& u_{g x}=\bar{g}_{x}+b_{g x}+n_{g x}+e_{a x} \bar{g}_{x}+\Delta_{x z} \bar{g}_{y}-\Delta_{x y} \bar{g}_{z} \\
& u_{g y}=\bar{g}_{y}+b_{g y}+n_{g y}+e_{a y} \bar{g}_{y}-\Delta_{y z} \bar{g}_{x}+\Delta_{y x} \bar{g}_{z} \\
& u_{g z}=\bar{g}_{z}+b_{g z}+n_{g z}+e_{a z} \bar{g}_{z}+\Delta_{z y} \bar{g}_{x}-\Delta_{z x} \bar{g}_{y} .
\end{aligned}
$$

According to scalar method of calibration [10, 11] let us sum of squared of normalized accelerometer's output signals as following below

$$
\begin{aligned}
& u_{g x}^{2}+u_{g y}^{2}+u_{g z}^{2}= \\
& {\left[\bar{g}_{x}+\left(b_{g x}+n_{g x}+e_{a x} \bar{g}_{x}+\Delta_{x z} \bar{g}_{y}-\Delta_{x y} \bar{g}_{z}\right)\right]^{2}+} \\
& {\left[\bar{g}_{y}+\left(b_{g y}+n_{g y}+e_{a y} \bar{g}_{y}-\Delta_{y z} \bar{g}_{x}+\Delta_{y x} \bar{g}_{z}\right)\right]^{2}+} \\
& {\left[\bar{g}_{z}+\left(b_{g z}+n_{g z}+e_{a z} \bar{g}_{z}+\Delta_{z y} \bar{g}_{x}-\Delta_{z x} \bar{g}_{y}\right)\right]^{2} .}
\end{aligned}
$$

It is necessary to calculate the scalar value of measuring vector and compare it to the known scalar value of measurable vector. For that let us remove of brackets in right side:

$$
\begin{aligned}
& u_{g x}^{2}+u_{g y}^{2}+u_{g z}^{2}=\bar{g}_{x}^{2}+ \\
& 2 \bar{g}_{x}\left(b_{g x}+n_{g x}+e_{a x} \bar{g}_{x}+\Delta_{x z} \bar{g}_{y}-\Delta_{x y} \bar{g}_{z}\right)+ \\
& +\left(b_{g x}+n_{g x}+e_{a x} \bar{g}_{x}+\Delta_{x z} \bar{g}_{y}-\Delta_{x y} \bar{g}_{z}\right)^{2}+\bar{g}_{y}^{2}+ \\
& 2 \bar{g}_{y}\left(b_{g y}+n_{g y}+e_{a y} \bar{g}_{y}-\Delta_{y z} \bar{g}_{x}+\Delta_{y x} \bar{g}_{z}\right)+ \\
& \left(b_{g y}+n_{g y}+e_{a y} \bar{g}_{y}-\Delta_{y z} \bar{g}_{x}+\Delta_{y x} \bar{g}_{z}\right)^{2}+\bar{g}_{z}^{2}+ \\
& 2 \bar{g}_{z}\left(b_{g z}+n_{g z}+e_{a z} \bar{g}_{z}+\Delta_{z y} \bar{g}_{x}-\Delta_{z x} \bar{g}_{y}\right)+ \\
& \left(b_{g z}+n_{g z}+e_{a z} \bar{g}_{z}+\Delta_{z y} \bar{g}_{x}-\Delta_{z x} \bar{g}_{y}\right)^{2} .
\end{aligned}
$$

As far as $\bar{g}_{x}^{2}+\bar{g}_{y}^{2}+\bar{g}_{z}^{2}=1$, and also ignoring values of the second order to the trifle like $(\ldots)^{2}$, for the triad of accelerometers will get

$$
\begin{aligned}
& \frac{1}{2}\left(u_{g x}^{2}+u_{g y}^{2}+u_{g z}^{2}-1\right)= \\
& \left(b_{g x}+n_{g x}\right) \bar{g}_{x}+\left(b_{g y}+n_{g y}\right) \bar{g}_{y}+\left(b_{g z}+n_{g z}\right) \bar{g}_{z}+ \\
& +e_{a x} \bar{g}_{x}^{2}+e_{a y} \bar{g}_{y}^{2}+e_{a z} \bar{g}_{z}^{2}+\delta_{a 1} \bar{g}_{x} \bar{g}_{y}+\delta_{a 2} \bar{g}_{x} \bar{g}_{z}+\delta_{a 3} \bar{g}_{y} \bar{g}_{z},
\end{aligned}
$$

Where

$$
\delta_{a 1}=\Delta_{x z}-\Delta_{y z}, \delta_{a 2}=\Delta_{z y}-\Delta_{x y}, \delta_{a 3}=\Delta_{y x}-\Delta_{z x} .
$$

For the triad of gyros $\left(\bar{\Omega}_{x}^{2}+\bar{\Omega}_{y}^{2}+\bar{\Omega}_{z}^{2}=1\right)$ analogically will get 


$$
\begin{aligned}
& \frac{1}{2}\left(u_{\Omega x}^{2}+u_{\Omega y}^{2}+u_{\Omega z}^{2}-1\right)= \\
& \left(b_{\Omega x}+n_{\Omega x}\right) \bar{\Omega}_{x}+\left(b_{\Omega y}+n_{\Omega y}\right) \bar{\Omega}_{y}+\left(b_{\Omega z}+n_{\Omega z}\right) \bar{\Omega}_{z}+ \\
& +e_{\omega x} \bar{\Omega}_{x}^{2}+e_{\omega y} \bar{\Omega}_{y}^{2}+e_{\omega z} \bar{\Omega}_{z}^{2}+\delta_{\omega 1} \bar{\Omega}_{x} \bar{\Omega}_{y}+\delta_{\omega 2} \bar{\Omega}_{x} \bar{\Omega}_{z}+\delta_{\omega 3} \bar{\Omega}_{y} \bar{\Omega}_{z} .
\end{aligned}
$$

Here

$$
\delta_{\omega 1}=\Delta_{x z}-\Delta_{y z}, \delta_{\omega 2}=\Delta_{z y}-\Delta_{x y}, \delta_{\omega 3}=\Delta_{y x}-\Delta_{z x} .
$$

Hence, the difference between the scalar value of the normalized measurable vector and his actual value that is equal to one, proportional to the errors of the inertial instrument cluster. Coefficients in this dependence are the normalized values of measurable acceleration $\bar{g}_{x}, \bar{g}_{y}, \bar{g}_{z}$ for accelerometers and angular rate $\bar{\Omega}_{x}, \bar{\Omega}_{y}, \bar{\Omega}_{z}$ for gyros, their exponential orders and compositions.

On the base of equations (7) and (8) let us build the algorithm of scalar method of quality monitoring for triad of accelerometers and gyros. For sampling time $t_{k}$ it is possible to establish following below predicates:

$$
\begin{gathered}
F_{0 g}\left(t_{k}\right)=\Lambda_{0 g}\left\{\frac{1}{2}\left(u_{g x}^{2}+u_{g y}^{2}+u_{g z}^{2}-1\right) \leq \lambda_{0 g}\right\}=\left\{\begin{array}{l}
1 \\
0
\end{array},\right. \\
F_{0 \Omega}\left(t_{k}\right)=\Lambda_{0 \Omega}\left\{\frac{1}{2}\left(u_{\Omega x}^{2}+u_{\Omega y}^{2}+u_{\Omega z}^{2}-1\right) \leq \lambda_{0 \Omega}\right\}=\left\{\begin{array}{l}
1 \\
0
\end{array} .\right.
\end{gathered}
$$

Here in right part a value ' 1 ' is mean an operable state of a triad of accelerometers or gyroscopes, a value ' 0 ' a his failure, $\lambda_{0 g}-$ a border value of function $\frac{1}{2}\left(u_{g x}^{2}+u_{g y}^{2}+u_{g z}^{2}-1\right)$. If the value of function $\left(u_{g x}^{2}+u_{g y}^{2}+u_{g z}^{2}-1\right)$ will not more than a value $2 \lambda_{0 g}$, therefore a triad of accelerometers has being in operable state. If not, therefore there is a failure. The same rule is valid for quality monitoring of gyros.

When the task of the quality monitoring is solved it is necessary to find a place and clear the reason of failure.

For that 18 unknown parameters should be found from equations (7) and (8). These 18 parameters are distorted of the inertial instrument cluster output signals. Six of them are differences of mounting misalignments angles of the devices.

According to scalar calibration of the inertial measurement unit we should in the gravity field to turn around certain direction at fixed angles and in every position get the normalized output signals. To solve the equations (7) and (8) it requires at least nine of the inertial instrument cluster position, so number of tests should be more or equal of nine. The fact is that in each one position of the inertial instrument cluster its output signals simultaneously have been measuring either gyroscopes or accelerometers, so the minimum number of positions in the two times less than the total number of required parame- ters.

Consider the equation (7) and (8) in matrix form for $n$ - testing operations or measurements:

$$
\mathbf{u}_{\mathrm{g}}=\mathbf{G} \cdot \mathbf{e}_{\mathrm{g}}, \quad \mathbf{u}_{\mathbf{\Omega}}=\mathbf{\Omega} \mathbf{e}_{\mathbf{\Omega}},
$$

where $\mathbf{u}_{\mathrm{a}}, \mathbf{u}_{\mathrm{g}}$ is a $n \times 1$ column vectors of the normalized inertial measurement unit output signals:

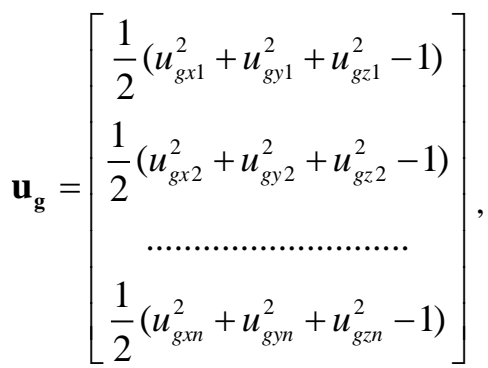

$$
\begin{aligned}
& \mathbf{u}_{\Omega}=\left[\begin{array}{c}
\frac{1}{2}\left(u_{\Omega x 1}^{2}+u_{\Omega y 1}^{2}+u_{\Omega z 1}^{2}-1\right) \\
\frac{1}{2}\left(u_{\Omega x 2}^{2}+u_{\Omega y 2}^{2}+u_{\Omega z 2}^{2}-1\right) \\
\ldots \ldots \ldots \ldots \ldots \ldots \ldots \ldots \ldots \\
\frac{1}{2}\left(u_{\Omega x n}^{2}+u_{\Omega y n}^{2}+u_{\Omega z n}^{2}-1\right)
\end{array}\right]
\end{aligned}
$$

$\mathbf{G}, \boldsymbol{\Omega}$ - is a $n \times 9$ matrixes of normalized projections of the acceleration $\vec{g}$ and turn rate $\vec{\Omega}$ of dimension:

$$
\mathbf{G}^{\mathbf{T}}=\left[\begin{array}{cccc}
\bar{g}_{x 1} & \bar{g}_{x 2} & \ldots & \bar{g}_{x n} \\
\bar{g}_{y 1} & \bar{g}_{y 2} & \ldots & \bar{g}_{y n} \\
\bar{g}_{z 1} & \bar{g}_{z 2} & \ldots & \bar{g}_{z n} \\
\bar{g}_{x 1}^{2} & \bar{g}_{x 2}^{2} & \ldots & \bar{g}_{x n}^{2} \\
\bar{g}_{y 1}^{2} & \bar{g}_{y 2}^{2} & \ldots & \bar{g}_{y n}^{2} \\
\bar{g}_{z 1}^{2} & \bar{g}_{z 2}^{2} & \ldots & \bar{g}_{z n}^{2} \\
\bar{g}_{x 1} \bar{g}_{y 1} & \bar{g}_{x 2} \bar{g}_{y 2} & \ldots & \bar{g}_{x n} \bar{g}_{y n} \\
\bar{g}_{x 1} \bar{g}_{z 1} & \bar{g}_{x 2} \bar{g}_{z 2} & \ldots & \bar{g}_{x n} \bar{g}_{z n} \\
\bar{g}_{y 1} \bar{g}_{z 1} & \bar{g}_{y 2} \bar{g}_{z 2} & \ldots & \bar{g}_{y n} \bar{g}_{z n}
\end{array}\right] ;
$$


$\mathbf{e}_{\mathrm{g}}, \mathbf{e}_{\boldsymbol{\Omega}}$ - is a $9 \times 1$ column vectors of unknown parameters

$$
\mathbf{e}_{\mathbf{g}}=\left[\begin{array}{c}
b_{g x}+n_{g x} \\
b_{g y}+n_{g y} \\
b_{g z}+n_{g z} \\
e_{a x} \\
e_{a y} \\
e_{a z} \\
\delta_{a 1} \\
\delta_{a 2} \\
\delta_{a 2}
\end{array}\right] ; \mathbf{e}_{\Omega}=\left[\begin{array}{c}
b_{\Omega x}+n_{\Omega x} \\
b_{\Omega y}+n_{\Omega y} \\
b_{\Omega z}+n_{\Omega z} \\
e_{\omega x} \\
e_{\omega y} \\
e_{\omega z} \\
\delta_{\omega 1} \\
\delta_{\omega 2} \\
\delta_{\omega 3}
\end{array}\right] .
$$

Solving the matrix equation (9) by least-squares method, we obtain:

$$
\begin{aligned}
& \hat{\mathbf{e}}_{\mathrm{g}}=\left(\mathbf{G}^{\mathrm{T}} \mathbf{G}\right)^{-1} \mathbf{G}^{\mathrm{T}} \mathbf{u}_{\mathrm{g}}, \\
& \hat{\mathbf{e}}_{\boldsymbol{\Omega}}=\left(\boldsymbol{\Omega}^{\mathrm{T}} \boldsymbol{\Omega}\right)^{-1} \boldsymbol{\Omega}^{\mathrm{T}} \mathbf{u}_{\boldsymbol{\Omega}},
\end{aligned}
$$

where $\hat{\mathbf{e}}_{\boldsymbol{\Omega}}, \hat{\mathbf{e}}_{\mathrm{g}}-$ is an estimating values of the unknown parameters of inertial measurement unit.

Thanks to the least squares method the results are smoothing, and as long as average of distribution is equal to zero

$$
M\left\{n_{x}\right\}=M\left\{n_{y}\right\}=M\left\{n_{z}\right\}=0,
$$

then estimated values $\hat{\mathbf{e}}_{\Omega}, \hat{\mathbf{e}}_{\mathrm{g}}$ will not have a random noise:

$$
\hat{\mathbf{e}}_{\mathbf{g}}=\left[\begin{array}{c}
\hat{b}_{g x} \\
\hat{b}_{g y} \\
\hat{b}_{g z} \\
\hat{e}_{a x} \\
\hat{e}_{a y} \\
\hat{e}_{a z} \\
\hat{\delta}_{a 1} \\
\hat{\delta}_{a 2} \\
\hat{\delta}_{a 3}
\end{array}\right], \hat{\mathbf{e}}_{\Omega}=\left[\begin{array}{l}
\hat{b}_{\Omega x} \\
\hat{b}_{\Omega y} \\
\hat{b}_{\Omega z} \\
\hat{e}_{\omega x} \\
\hat{e}_{\omega y} \\
\hat{e}_{\omega z} \\
\hat{\delta}_{\omega 1} \\
\hat{\delta}_{\omega 2} \\
\hat{\delta}_{\omega 3}
\end{array}\right] .
$$

According to introduced relationships (5) we can calculate estimations of $B_{\omega(a) x}, B_{\omega(a) y}, B_{\omega(a) z} \quad$ and $E_{\omega(a) x}, E_{\omega(a) y}, E_{\omega(a) z}$ as follows:

$$
\begin{aligned}
& \hat{B}_{a j}=\hat{b}_{g j} S_{a j} g ; \quad \hat{E}_{a j}=\hat{e}_{a j} S_{a j} ; \\
& \hat{B}_{\omega j}=\hat{b}_{\Omega j} S_{\omega j} \Omega ; \quad \hat{E}_{\omega j}=\hat{e}_{\omega j} S_{\omega j} .
\end{aligned}
$$

When estimated values (12) are calculated, it is possible to use following set of predicates, which are expressed the algorithm of diagnostics of gyroscopes triad on stationary base:

$$
\begin{gathered}
F_{1 \Omega 2}\left(t_{k}\right)=\Lambda_{\Omega}\left\{\left|\hat{B}_{\omega x}-B_{\omega x}\right| \leq \lambda_{1 \Omega}\right\}=\left\{\begin{array}{l}
1 ; \\
0
\end{array}\right. \\
F_{2 \Omega}\left(t_{k}\right)=\Lambda_{\Omega}\left\{\left|\hat{B}_{\omega y}-B_{\omega y}\right| \leq \lambda_{2 \Omega}\right\}=\left\{\begin{array}{l}
1 \\
0
\end{array}\right. \\
F_{1 \Omega 2}\left(t_{k}\right)=\Lambda_{\Omega}\left\{\left|\hat{B}_{\omega x}-B_{\omega x}\right| \leq \lambda_{1 \Omega}\right\}=\left\{\begin{array}{l}
1 \\
0
\end{array} ;\right. \\
F_{4 \Omega(}\left(t_{k}\right)=\Lambda_{\Omega}\left\{\left|\hat{E}_{\omega x}-E_{\omega x}\right| \leq \lambda_{4 \Omega}\right\}=\left\{\begin{array}{l}
1 \\
0
\end{array} ;\right. \\
F_{5 \Omega}\left(t_{k}\right)=\Lambda_{\Omega}\left\{\left|\hat{E}_{\omega y}-E_{\omega y}\right| \leq \lambda_{5 \Omega}\right\}=\left\{\begin{array}{l}
1 \\
0
\end{array} ;\right. \\
F_{6 \Omega(}\left(t_{k}\right)=\Lambda_{\Omega}\left\{\left|\hat{E}_{\omega z}-E_{\omega z}\right| \leq \lambda_{6 \Omega}\right\}=\left\{\begin{array}{l}
1 \\
0
\end{array} ;\right. \\
F_{7 \Omega \Omega}\left(t_{k}\right)=\Lambda_{\Omega}\left\{\left|\hat{\delta}_{\omega 1}-\delta_{\omega 1}\right| \leq \lambda_{\Omega \Omega}\right\}=\left\{\begin{array}{l}
1 \\
0
\end{array} ;\right. \\
F_{8 \Omega(}\left(t_{k}\right)=\Lambda_{\Omega}\left\{\left|\hat{\delta}_{\omega 2}-\delta_{\omega 2}\right| \leq \lambda_{8 \Omega}\right\}=\left\{\begin{array}{l}
1 \\
0
\end{array} ;\right. \\
F_{9 \Omega 2}\left(t_{k}\right)=\Lambda_{\Omega}\left\{\left|\hat{\delta}_{\omega 3}-\delta_{\omega 3}\right| \leq \lambda_{9 \Omega}\right\}=\left\{\begin{array}{l}
1 \\
0
\end{array} .\right.
\end{gathered}
$$

Here $\lambda_{1 \Omega}, \lambda_{2 \Omega}, \lambda_{3 \Omega}$ - border values of gyro biases, $\lambda_{4 \Omega}, \lambda_{5 \Omega}, \lambda_{6 \Omega}$ - border values of gyro scale factor errors, $\lambda_{7 \Omega}, \lambda_{8 \Omega}, \lambda_{9 \Omega}$ - border values of gyro mounting misalignments. If the difference between calculated values $\hat{\mathbf{e}}$ will not more than a values $\pm \lambda_{i}$, therefore a triad of gyroscopes has being in operable state. If not, therefore there is a failure. The number of $\hat{\mathbf{e}}$, which are excited out of value $\pm \lambda_{i}$, indicate not only what gyro is failure, but also indicate a reason of failure: excessing of real biases, scale factor errors or mounting misalignments to their nominal values.

The scheme of scalar method of fault diagnosis systems of IMU is depicted in the Fig. 5. Here numbers 1, 2, 3 is shown the gyro's failures via biases discrepancy, numbers 4,5,6 - gyro's failures via scale factor errors discrepancy and numbers 7,8,9 - gyro's failures via mounting misalignments discrepancy.

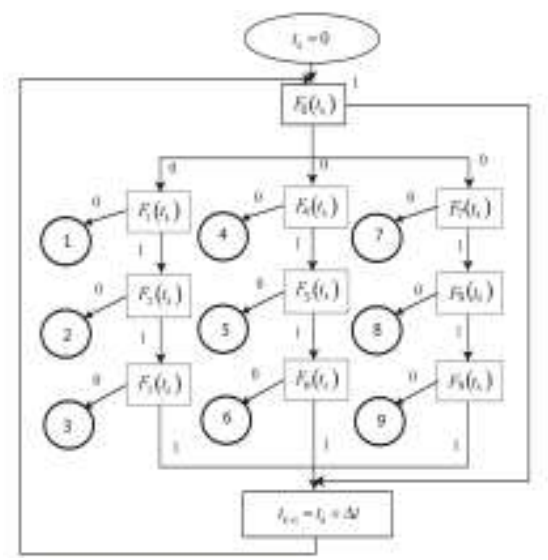

Fig.5. Scheme of scalar method of fault diagnosis systems of gyro's triad IMU 


\section{The Scalar Diagnostics ON THE Moving Base}

Let us consider scalar diagnostics on the moving base. Using initial equations (1) and (2) we can receive following relations for gyro cluster

$$
\begin{aligned}
& U_{\omega x}=B_{\omega x}+\left(S_{\omega x}+E_{\omega x}\right) \omega_{x}+S_{\omega x} \Delta_{x z} \omega_{y}-S_{\omega x} \Delta_{x y} \omega_{z}+w_{\omega x} \\
& U_{\omega y}=B_{\omega y}+\left(S_{\omega y}+E_{\omega y}\right) \omega_{y}-S_{\omega y} \Delta_{y z} \omega_{x}+S_{\omega y} \Delta_{y x} \omega_{z}+w_{\omega y} \\
& U_{\omega z}=B_{\omega z}+\left(S_{\omega z}+E_{\omega z}\right) \omega_{z}+S_{\omega z} \Delta_{z y} \omega_{x}-S_{\omega z} \Delta_{z x} \omega_{y}+w_{\omega z},
\end{aligned}
$$

and for accelerometer's cluster

$$
\begin{aligned}
& U_{a x}=B_{a x}+\left(S_{a x}+E_{a x}\right) a_{x}+S_{a x} \Delta_{x z} a_{y}-S_{a x} \Delta_{x y} a_{z}+w_{a x} \\
& U_{a y}=B_{a y}+\left(S_{a y}+E_{a y}\right) a_{y}-S_{a y} \Delta_{y z} a_{x}+S_{a y} \Delta_{y x} a_{z}+w_{a y} \\
& U_{a z}=B_{a z}+\left(S_{a z}+E_{a z}\right) a_{z}+S_{a z} \Delta_{z y} a_{x}-S_{a z} \Delta_{z x} a_{y}+w_{a z}
\end{aligned}
$$

Fig. 6 and Fig. 7 shows examples of output signals of gyroscopes and accelerometers of IMU with USB-port [13] on the moving base (takeoff with sharp turn).
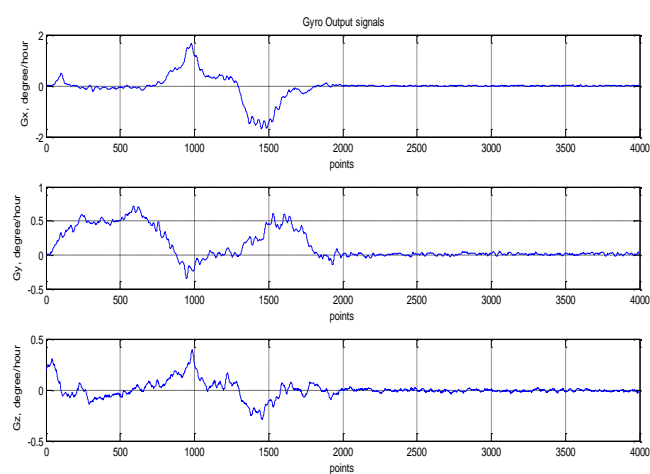

Fig.6. Output signals of gyroscopes ADXRS22295 (Gx and Gy) and ADXRS300 $(\mathrm{Gz})$ on the moving base.
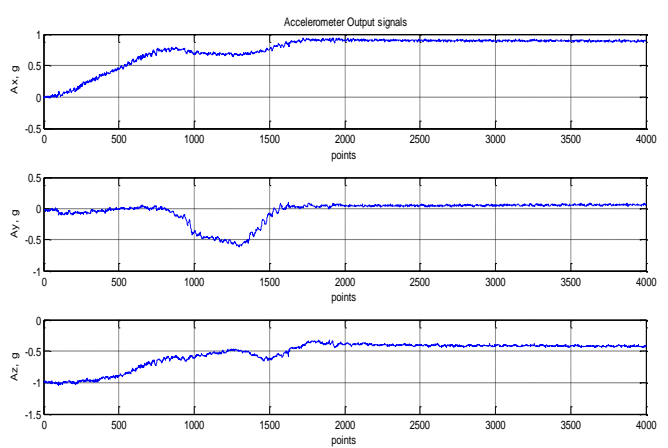

Fig.7. Output signals of accelerometers ADXL202 (Ax, Ay and Az) on the moving base.

According to scalar diagnostics method let's divide every expression of output signal of accelerometer on corresponding scale factor and vector's module $a=\sqrt{a_{x}^{2}+a_{y}^{2}+a_{z}^{2}}$ and every expression of output gyro signal on corresponding scale factor and vector's module $\omega=\sqrt{\omega_{x}^{2}+\omega_{y}^{2}+\omega_{z}^{2}}$.

New denotations of dimensionless output signals and values of right parts will be as follows:

$$
\begin{gathered}
u_{a j}=\frac{U_{a j}}{S_{a j} a} ; \quad \bar{a}_{j}=\frac{a_{j}}{a} ; b_{a j}=\frac{B_{a j}}{S_{a j} a} ; \\
e_{a j}=\frac{E_{a j}}{S_{a j}} ; n_{a j}=\frac{n_{a j}}{S_{a j} a} ; \quad u_{\omega j}=\frac{U_{\omega j}}{S_{\omega j} \omega} ; \\
\bar{\omega}_{j}=\frac{\omega_{j}}{\omega} ; \quad b_{\omega j}=\frac{B_{\omega j}}{S_{\omega j} \omega} ; e_{\omega j}=\frac{E_{\omega j}}{S_{\omega j}} ; \\
n_{\omega j}=\frac{n_{\omega j}}{S_{\omega j} \omega} .
\end{gathered}
$$

Here $j=x, y, z$.

On the stationary base we should to use magnitude of gravity vector $g$ and Earth's rate $\Omega$. But how we can get current values of $a_{x}, a_{y}, a_{z}$ and $\omega_{x}, \omega_{y}, \omega_{z}$ on the moving base for calculations of $a=\sqrt{a_{x}^{2}+a_{y}^{2}+a_{z}^{2}}$ and $\omega=\sqrt{\omega_{x}^{2}+\omega_{y}^{2}+\omega_{z}^{2}}$ ?

Solving equations (1) and (2), we can receive estimated values $\hat{\omega}_{x}, \hat{\omega}_{y}, \hat{\omega}_{z}$ :

$$
\left[\begin{array}{c}
\hat{\omega}_{x} \\
\hat{\omega}_{y} \\
\hat{\omega}_{z}
\end{array}\right]=\mathbf{K}_{\omega}^{-1}\left(\left[\begin{array}{c}
U_{\omega x} \\
U_{\omega y} \\
U_{\omega z}
\end{array}\right]-\left[\begin{array}{c}
\hat{B}_{\omega x} \\
\hat{B}_{\omega y} \\
\hat{B}_{\omega z}
\end{array}\right]\right),
$$

Also estimated value of the accelerations $\hat{a}_{x}, \hat{a}_{y}, \hat{a}_{z}$ could be calculated as

$$
\left[\begin{array}{l}
\hat{a}_{x} \\
\hat{a}_{y} \\
\hat{a}_{z}
\end{array}\right]=\mathbf{K}_{\mathbf{a}}^{-\mathbf{1}}\left(\left[\begin{array}{c}
U_{a x} \\
U_{a y} \\
U_{a z}
\end{array}\right]-\left[\begin{array}{c}
\hat{B}_{a x} \\
\hat{B}_{a y} \\
\hat{B}_{a z}
\end{array}\right]\right) .
$$

Now we can receive $\hat{a}=\sqrt{\hat{a}_{x}^{2}+\hat{a}_{y}^{2}+\hat{a}_{z}^{2}}$ and $\hat{\omega}=\sqrt{\hat{\omega}_{x}^{2}+\hat{\omega}_{y}^{2}+\hat{\omega}_{z}^{2}}$.

As far as on the moving base for the triad of gyros $\bar{\omega}_{x}^{2}+\bar{\omega}_{y}^{2}+\bar{\omega}_{z}^{2}=1$ we will have following equation:

$$
\begin{aligned}
& \frac{1}{2}\left(u_{\omega x}^{2}+u_{\omega y}^{2}+u_{\omega z}^{2}-1\right)= \\
& \left(b_{\omega x}+n_{\omega x}\right) \bar{\omega}_{x}+\left(b_{\omega y}+n_{\omega y}\right) \bar{\omega}_{y}+\left(b_{\omega z}+n_{\omega z}\right) \bar{\omega}_{z}+ \\
& +e_{\omega x} \bar{\omega}_{x}^{2}+e_{\omega y} \bar{\omega}_{y}^{2}+e_{\omega z} \bar{\omega}_{z}^{2}+ \\
& \delta_{\omega 1} \bar{\omega}_{x} \bar{\omega}_{y}+\delta_{\omega 2} \bar{\omega}_{x} \bar{\omega}_{z}+\delta_{\omega 3} \bar{\omega}_{y} \bar{\omega}_{z} .
\end{aligned}
$$


For the triad of accelerometers $\bar{a}_{x}^{2}+\bar{a}_{y}^{2}+\bar{a}_{z}^{2}=1$ will get us similar equation:

$$
\begin{aligned}
& \frac{1}{2}\left(u_{a x}^{2}+u_{a y}^{2}+u_{a z}^{2}-1\right)= \\
& \left(b_{a x}+n_{a x}\right) \bar{a}_{x}+\left(b_{a y}+n_{a y}\right) \bar{a}_{y}+\left(b_{a z}+n_{a z}\right) \bar{a}_{z}+ \\
& +e_{a x} \bar{a}_{x}^{2}+e_{a y} \bar{a}_{y}^{2}+e_{a z} \bar{a}_{z}^{2}+ \\
& \delta_{a 1} \bar{a}_{x} \bar{a}_{y}+\delta_{a 2} \bar{a}_{x} \bar{a}_{z}+\delta_{a 3} \bar{a}_{y} \bar{a}_{z} .
\end{aligned}
$$

Hence, the difference between the scalar value of the normalized measurable vector and his actual value that is equal to one, proportional to the errors of the inertial instrument cluster. Coefficients in this dependence are the normalized values of measurable acceleration $\bar{a}_{x}, \bar{a}_{y}, \bar{a}_{z}$ for accelerometers and angular rate $\bar{\omega}_{x}, \bar{\omega}_{y}, \bar{\omega}_{z}$ for gyros, their exponential orders and compositions.

Analogically to algorithm of scalar monitoring on the stationary base, from equations (19) and (20) we can build the algorithm of scalar method of quality monitoring for triad of accelerometers and gyros for moving base. For sampling time $t_{k}$ it is possible to establish following below predicates:

$$
\begin{aligned}
& F_{0 a}\left(t_{k}\right)=\Lambda_{0 a}\left\{\frac{1}{2}\left(u_{a x}^{2}+u_{a y}^{2}+u_{a z}^{2}-1\right) \leq \lambda_{0 a}\right\}=\left\{\begin{array}{l}
1, \\
0
\end{array},\right. \\
& F_{0 \omega}\left(t_{k}\right)=\Lambda_{0}\left\{\frac{1}{2}\left(u_{\omega x}^{2}+u_{\omega y}^{2}+u_{\omega z}^{2}-1\right) \leq \lambda_{0 \omega}\right\}=\left\{\begin{array}{l}
1 \\
0
\end{array} .\right.
\end{aligned}
$$

Here in right part a value ' 1 ' is mean an operable state of a triad of accelerometers or gyroscopes, a value ' 0 ' a his failure, $\lambda_{0 a}-\mathrm{a}$ border value of function $\frac{1}{2}\left(u_{a x}^{2}+u_{a y}^{2}+u_{a z}^{2}-1\right)$. If the value of function $\left(u_{a x}^{2}+u_{a y}^{2}+u_{a z}^{2}-1\right)$ will not more than a value $2 \lambda_{0 g}$, therefore a triad of accelerometers has being in operable state. If not, therefore there is a failure. The same rule is valid for quality monitoring of gyros.

When the task of the quality monitoring is solved it is necessary to find a place and clear the reason of failure.

For that 18 unknown parameters should be found from equations (19) and (20). These 18 parameters are distorted of the inertial instrument cluster output signals. Six of them are differences of mounting misalignments angles of the devices.

Consider the equation (19) and (20) in matrix-block form:

$$
\begin{aligned}
& \mathbf{u}_{\mathrm{a}}=\mathbf{A} \cdot \mathbf{e}_{\mathrm{a}}, \\
& \mathbf{u}_{\omega}=\omega \cdot \mathbf{e}_{\omega},
\end{aligned}
$$

where $\mathbf{u}_{\mathrm{a}}, \mathbf{u}_{\boldsymbol{\Omega}}$ is a $n \times 1$ column vectors of the normalized inertial measurement unit output signals:

$$
\begin{aligned}
& \mathbf{u}_{\mathbf{a}}=\left[\begin{array}{c}
\frac{1}{2}\left(u_{a x 1}^{2}+u_{a y 1}^{2}+u_{a z 1}^{2}-1\right) \\
\frac{1}{2}\left(u_{a x 2}^{2}+u_{a y 2}^{2}+u_{a z 2}^{2}-1\right) \\
\ldots \ldots \ldots \ldots \ldots \ldots \ldots \ldots \ldots \ldots \ldots \ldots \ldots \ldots \ldots \ldots \\
\frac{1}{2}\left(u_{a x n}^{2}+u_{a y n}^{2}+u_{a z n}^{2}-1\right)
\end{array}\right], \\
& \mathbf{u}_{\omega}=\left[\begin{array}{c}
\frac{1}{2}\left(u_{\omega x 1}^{2}+u_{\omega y 1}^{2}+u_{\omega z 1}^{2}-1\right) \\
\frac{1}{2}\left(u_{\omega x 2}^{2}+u_{\omega y 2}^{2}+u_{\omega z 2}^{2}-1\right) \\
\cdots \ldots \ldots \ldots \ldots \ldots \ldots \ldots \ldots \ldots \ldots \ldots \ldots \ldots \ldots \\
\frac{1}{2}\left(u_{\omega x n}^{2}+u_{\omega y n}^{2}+u_{\omega z n}^{2}-1\right)
\end{array}\right],
\end{aligned}
$$

A, $\boldsymbol{\omega}$ - is a $n \times 9$ matrixes of normalized projections of the acceleration $\vec{a}$ and turn rate $\vec{\omega}$ of dimension:

$$
\begin{aligned}
& \mathbf{A}^{\mathbf{T}}=\left[\begin{array}{cccc}
\bar{a}_{x 1} & \bar{a}_{x 2} & \ldots & \bar{a}_{x n} \\
\bar{a}_{y 1} & \bar{a}_{y 2} & \ldots & \bar{a}_{y n} \\
\bar{a}_{z 1} & \bar{a}_{z 2} & \ldots & \bar{a}_{z n} \\
\bar{a}_{x 1}^{2} & \bar{a}_{x 2}^{2} & \ldots & \bar{a}_{x n}^{2} \\
\bar{a}_{y 1}^{2} & \bar{a}_{y 2}^{2} & \ldots & \bar{a}_{y n}^{2} \\
\bar{a}_{z 1}^{2} & \bar{a}_{z 2}^{2} & \ldots & \bar{a}_{z n}^{2} \\
\bar{a}_{x 1} \bar{a}_{y 1} & \bar{a}_{x 2} \bar{a}_{y 2} & \ldots & \bar{a}_{x n} \bar{a}_{y n} \\
\bar{a}_{x 1} \bar{a}_{z 1} & \bar{a}_{x 2} \bar{a}_{z 2} & \ldots & \bar{a}_{x n} \bar{a}_{z n} \\
\bar{a}_{y 1} \bar{a}_{z 1} & \bar{a}_{y 2} \bar{a}_{z 2} & \ldots & \bar{a}_{y n} \bar{a}_{z n}
\end{array}\right] ; \\
& \boldsymbol{\omega}^{\mathbf{T}}=\left[\begin{array}{cccc}
\bar{\omega}_{x 1} & \bar{\omega}_{x 2} & \ldots & \bar{\omega}_{x n} \\
\bar{\omega}_{y 1} & \bar{\omega}_{y 2} & \ldots & \bar{\omega}_{y n} \\
\bar{\omega}_{z 1} & \bar{\omega}_{z 2} & \ldots & \bar{\omega}_{z n} \\
\bar{\omega}_{x 1}^{2} & \bar{\omega}_{x 2}^{2} & \ldots & \bar{\omega}_{x n}^{2} \\
\bar{\omega}_{y 1}^{2} & \bar{\omega}_{y 2}^{2} & \ldots & \bar{\omega}_{y n}^{2} \\
\bar{\omega}_{z 1}^{2} & \bar{\omega}_{z 2}^{2} & \ldots & \bar{\omega}_{z n}^{2} \\
\bar{\omega}_{x 1} \bar{\omega}_{y 1} & \bar{\omega}_{x 2} \bar{\omega}_{y 2} & \ldots & \bar{\omega}_{x n} \bar{\omega}_{y n} \\
\bar{\omega}_{x 1} \bar{\omega}_{z 1} & \bar{\omega}_{x 2} \bar{\omega}_{z 2} & \ldots & \bar{\omega}_{x n} \bar{\omega}_{z n} \\
\bar{\omega}_{y 1} \bar{\omega}_{z 1} & \bar{\omega}_{y 2} \bar{\omega}_{z 2} & \ldots & \bar{\omega}_{y n} \bar{\omega}_{z n}
\end{array}\right] ;
\end{aligned}
$$

$\mathbf{e}_{\mathrm{a}}, \mathbf{e}_{\boldsymbol{\omega}}$ - is a $9 \times 1$ column vectors of unknown parameters

$$
\mathbf{e}_{\mathbf{a}}=\left[\begin{array}{c}
b_{a x}+n_{a x} \\
b_{a y}+n_{a y} \\
b_{a z}+n_{a z} \\
e_{a x} \\
e_{a y} \\
e_{a z} \\
\delta_{a 1} \\
\delta_{a 2} \\
\delta_{a 2}
\end{array}\right] ; \mathbf{e}_{\omega}=\left[\begin{array}{c}
b_{\omega x}+n_{\omega x} \\
b_{\omega y}+n_{\omega y} \\
b_{\omega z}+n_{\omega z} \\
e_{\omega x} \\
e_{\omega y} \\
e_{\omega z} \\
\delta_{\omega 1} \\
\delta_{\omega 2} \\
\delta_{\omega 3}
\end{array}\right] .
$$


Solving the matrix equation (23) by least-squares method, we obtain:

$$
\begin{aligned}
& \hat{\mathbf{e}}_{\mathrm{a}}=\left(\mathbf{A}^{\mathrm{T}} \mathbf{A}\right)^{-1} \mathbf{A}^{\mathrm{T}} \mathbf{u}_{\mathrm{a}}, \\
& \hat{\mathbf{e}}_{\omega}=\left(\omega^{\mathrm{T}} \omega\right)^{-1} \omega^{\mathrm{T}} \mathbf{u}_{\omega}
\end{aligned}
$$

where $\hat{\mathbf{e}}_{\mathbf{a}}, \hat{\mathbf{e}}_{\mathbf{\omega}}$ - is an estimating values of the unknown parameters of inertial measurement unit.

Thanks to the least squares method the results are smoothing, and as long as average of distribution is equal to zero

$$
M\left\{n_{x}\right\}=M\left\{n_{y}\right\}=M\left\{n_{z}\right\}=0,
$$

then estimated values $\hat{\mathbf{e}}_{\mathbf{a}}, \hat{\mathbf{e}}_{\boldsymbol{\omega}}$ will not have a random noise:

$$
\hat{\mathbf{e}}_{\mathbf{a}}=\left[\begin{array}{c}
\hat{b}_{a x} \\
\hat{b}_{a y} \\
\hat{b}_{a z} \\
\hat{e}_{a x} \\
\hat{e}_{a y} \\
\hat{e}_{a z} \\
\hat{\delta}_{a 1} \\
\hat{\delta}_{a 2} \\
\hat{\delta}_{a 2}
\end{array}\right] ; \hat{\mathbf{e}}_{\omega}=\left[\begin{array}{c}
\hat{b}_{\omega x} \\
\hat{b}_{\omega y} \\
\hat{b}_{\omega z} \\
\hat{e}_{\omega x} \\
\hat{e}_{\omega y} \\
\hat{e}_{\omega z} \\
\hat{\delta}_{\omega 1} \\
\hat{\delta}_{\omega 2} \\
\hat{\delta}_{\omega 3}
\end{array}\right] .
$$

According to introduced relationships (16) we can calculate estimations of $B_{\omega(a) x}, B_{\omega(a) y}, B_{\omega(a) z} \quad$ and $E_{\omega(a) x}, E_{\omega(a) y}, E_{\omega(a) z}$ on moving base as follows:

$$
\begin{aligned}
& \hat{B}_{a j}=\hat{b}_{g j} S_{a j} a ; \quad \hat{E}_{a j}=\hat{e}_{a j} S_{a j} \\
& \hat{B}_{\omega j}=\hat{b}_{\Omega j} S_{\omega j} \omega ; \quad \hat{E}_{\omega j}=\hat{e}_{\omega j} S_{\omega j} .
\end{aligned}
$$

And algorithm of scalar method of diagnostics of accelerometers triad will correspond to predicates

$$
\begin{aligned}
& F_{1 a}\left(t_{k}\right)=\Lambda_{a}\left\{\left|\hat{B}_{a x}-B_{a x}\right| \leq \lambda_{1 a}\right\}=\left\{\begin{array}{l}
1 \\
0
\end{array}\right. \\
& F_{2 a}\left(t_{k}\right)=\Lambda_{a}\left\{\left|\hat{B}_{a y}-B_{a y}\right| \leq \lambda_{2 a}\right\}=\left\{\begin{array}{l}
1 \\
0
\end{array} ;\right. \\
& F_{3 a}\left(t_{k}\right)=\Lambda_{a}\left\{\left|\hat{B}_{a z}-B_{a z}\right| \leq \lambda_{3 a}\right\}=\left\{\begin{array}{l}
1 \\
0
\end{array} ;\right. \\
& F_{4 a}\left(t_{k}\right)=\Lambda_{a}\left\{\left|\hat{E}_{a x}-E_{a x}\right| \leq \lambda_{4 a}\right\}=\left\{\begin{array}{l}
1 \\
0
\end{array} ;\right.
\end{aligned}
$$

$$
\begin{aligned}
& F_{5 a}\left(t_{k}\right)=\Lambda_{a}\left\{\left|\hat{E}_{a y}-E_{a y}\right| \leq \lambda_{5 a}\right\}=\left\{\begin{array}{l}
1 \\
0
\end{array} ;\right. \\
& F_{6 a}\left(t_{k}\right)=\Lambda_{a}\left\{\left|\hat{E}_{a z}-E_{a z}\right| \leq \lambda_{6 a}\right\}=\left\{\begin{array}{l}
1 \\
0
\end{array} ;\right. \\
& F_{7 a}\left(t_{k}\right)=\Lambda_{a}\left\{\left|\hat{\delta}_{a 1}-\delta_{a 1}\right| \leq \lambda_{7 a}\right\}=\left\{\begin{array}{l}
1 \\
0
\end{array} ;\right. \\
& F_{8 a}\left(t_{k}\right)=\Lambda_{a}\left\{\left|\hat{\delta}_{a 2}-\delta_{a 2}\right| \leq \lambda_{8 a}\right\}=\left\{\begin{array}{l}
1 \\
0
\end{array}\right. \\
& F_{9 a}\left(t_{k}\right)=\Lambda_{a}\left\{\left|\hat{\delta}_{a 3}-\delta_{a 3}\right| \leq \lambda_{9 a}\right\}=\left\{\begin{array}{l}
1 \\
0
\end{array} .\right.
\end{aligned}
$$

Here $\lambda_{1 a}, \lambda_{2 a}, \lambda_{3 a}$ - border values of accelerometers biases, $\lambda_{4 a}, \lambda_{5 a}, \lambda_{6 a}$ - border values of accelerometers scale factor errors, $\lambda_{7 a}, \lambda_{8 a}, \lambda_{9 a}$ - border values of accelerometers mounting misalignments. If the difference between calculated values $\hat{\mathbf{e}}$ will not more than a values $\pm \lambda_{i}$, therefore a triad of gyroscopes has being in operable state. If not, therefore there is a failure. The number of $\hat{\mathbf{e}}$ which are excited out of value $\pm \lambda_{i}$, indicate not only what gyro is failure, but also indicate a reason of failure: excessing of real biases, scale factor errors or mounting misalignments to their nominal values.

\section{CONCLUSIONS}

In this paper we have proposed a new method of fault diagnosis of Strapdown Inertial Navigation Systems. The scalar calibration method is a base of the scalar method of quality monitoring and diagnostics. Algorithms of fault diagnosis systems are developed in accordance with scalar calibration method. In result of quality monitoring algorithm verification is implemented the working capacity monitoring of IMU. A failure element determination is based in diagnostics algorithm verification and after that the reason of such failure is cleared.

The process of verifications consists of comparison of the calculated estimations of biases, scale factor errors and misalignments angles of sensors to their data sheet certificate, which kept in internal memory of computer. In result of such comparison the conclusion for working capacity of each one IMU sensor can be made and also the failure sensor can be determined.

\section{ACKNOWLEDGEMENTS}

I am thankful to Mr. Bob Sulouff, Vice-President of Analog Devices Inc. ${ }^{\circledR}$ and his team for great support and samples of ADI gyroscopes and accelerometers.

I am very grateful to Dr. Oleg Stepanov, Professor of St. Petersburg ITMO University for time in Ladoga and kind comments.

Also I am thankful to my parents and my family who have always believed me. 


\section{REFERENCES}

[1] V. Avrutov, N. Bouraou, Reliability and Fault Diagnosis. - LAP Lambert Academic Publishing, 2015. - 164 p. (ISBN 978-3-659-64879-3) - In Russian.

[2] D. Titterton, J. Weston, Strapdown Inertial Navigation Technology $-2^{\text {nd }}$ Edition, Institution of Electrical Engineers , UK, 2004 - 558 p.

[3] S.P. Dmitriyev, N.V. Kolesov, A.V. Osipov, Information Reliability, Monitoring and Diagnostics of Navigation Systems. - SPb: GNC RF - CSRI 'Electropribor', 2003. 207 p. (ISBN 5-900780-46-5) - In Russian.

[4] S.P. Dmitriyev, O.A. Stepanov, S.V. Shepel, "Nonlinear Filtering Methods: Application in INS Alignment," IEEE Transactions on Aerospace and Electronic Systems, Vol. 33, No. 1, 1997, pp. 260-271.

[5] A.S. Kulik, S.N. Firsov, Do Kuok Tuan, O.Y. Zlatkin, "A Fault Diagnosis of Strapdown Inertial Navigation System for unmanned aircraft". - Radio Electronics and Computer Systems, 2008, v.1 (28), pp.75-81. - In Russian.

[6] R.J. Patton, P.M. Frank, R.N. Clark, "Issues in fault diagnosis for dynamic systems". - Springer-Verlag, London, 2000.

[7] Puneet Kumar Singh and D. K. Chaturvedi, "Neural Network based Modeling and Simulation of Transformer Inrush Current". I.J. Intelligent Systems and Applications, 2012, 5, pp.1-7.

[8] K. A. Ellithy, K. A. El-Metwally, "Design of Decentralized Fuzzy Logic Load Frequency Controller. I.J. Intelligent Systems and Applications, 2012, 2, pp.66-75.

[9] Y. V. Bodyanskiy, O. K. Tyshchenko, D. S. Kopaliani, "An Extended Neo-Fuzzy Neuron and its Adaptive Learning Algorithm". I.J. Intelligent Systems and Applications, 2015, 02, pp.21-26.

[10] E. Izmailov, S. Lepe, A. Molchanov, E. Polikovsky, "Scalar method of calibration and balancing of Strapdown Inertial Navigation Systems". 15-th St. Petersburg International Conference on Integrated Navigation Systems. St. Petersburg, Russia, - State Research Center (CSRI) Elektropribor, 2008, pp.145-154. - In Russian.

[11] V. Avrutov, "On Scalar Calibration of an Inertial Instrument Cluster". Innovation and Technologies News, 2011, No. 2(11), pp. 22-30

[12] V. Avrutov, S. Golovach, T. Mazepa, "On Scalar Calibration of an Inertial Measurement Unit". 19-th St. Petersburg International Conference on Integrated Navigation Systems. St. Petersburg, Russia, 2012. - State Research Center (CSRI) Elektropribor, 2012, pp.117-121.

[13] V. Avrutov, I. Sturma, "Inertial Measurement Unit with USB-Port”. 19-th St. Petersburg International Conference on Integrated Navigation Systems. St. Petersburg, Russia, 2012. - State Research Center (CSRI) Elektropribor, 2012, pp.194-195.

\section{Authors' Profiles}

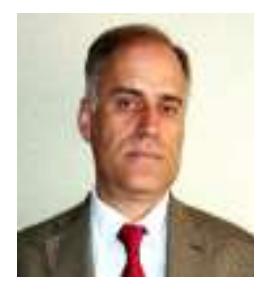

Vadim V. Avrutov has received the Dipl. Eng. degree in electro-mechanics from Kiev Polytechnic Institute in 1984 and Candidate of Technical Sciences degree in 1990. From 1984 to 1991 he was engaged in research of sea gyrocompasses. $\mathrm{He}$ is an inventor of first Ukrainian marine gyrocompass CRUISE - Patent of
Ukraine \#19719 dated 25.12.1997. Actually he is working as an Associate Professor of Instruments Making Faculty at National Technical University of Ukraine (Kiev Polytechnic Institute) with research interests in Testing \& Fault Diagnosis of Inertial Sensors and Navigation Technology.

Manuscript received March 09, 2015; revised May 18, 2015; accepted July 27, 2015.

How to cite this paper: Vadim V. Avrutov,"Scalar Diagnostics of the Inertial Measurement Unit", International Journal of Intelligent Systems and Applications (IJISA), vol.7, no.11, pp.1-9, 2015. DOI: 10.5815/ijisa.2015.11.01 